\title{
THE ABSOLUTELY CONTINUOUS SPECTRUM OF ONE-DIMENSIONAL SCHRÖDINGER OPERATORS
}

\author{
CHRISTIAN REMLING
}

\begin{abstract}
This paper deals with general structural properties of one-dimensional Schrödinger operators with some absolutely continuous spectrum. The basic result says that the $\omega$ limit points of the potential under the shift map are reflectionless on the support of the absolutely continuous part of the spectral measure. This implies an Oracle Theorem for such potentials and DenisovRakhmanov type theorems.

In the discrete case, for Jacobi operators, these issues were discussed in my recent paper [19]. The treatment of the continuous case in the present paper depends on the same basic ideas.
\end{abstract}

\section{INTRODUCTION}

This note discusses basic properties of one-dimensional Schrödinger operators

$$
H=-\frac{d^{2}}{d x^{2}}+V(x)
$$

with some absolutely continuous spectrum. It is a supplement to my recent paper [19]. In [19], I dealt with the discrete case exclusively. As one would expect, the basic ideas that were presented in [19] can also be used to analyze the continuous case. It is the purpose of this paper to give such a treatment; basically, this will be a matter of making the appropriate definitions.

Therefore, my general philosophy will be to keep this note brief. I will assume that the reader is familiar with at least the general outline of the discussion of [19] and only focus on those aspects where the extension to the continuous case is perhaps not entirely obvious. By the same token, I will not say much about related work here; please see again [19] for a fuller discussion.

Given a potential $V$, we will consider limit points $W$ under the shift $\left(S_{x} V\right)(t)=V(x+t)$, as $x \rightarrow \infty$. We will thus need a suitable topology

Date: October 29, 2007; revised: January 31, 2008.

Key words and phrases. Absolutely continuous spectrum, Schrödinger operator, reflectionless potential.

2000 Mathematics Subject Classification. Primary 34L40 81Q10. 
on a suitable space of potentials. This will naturally lead us to consider generalized Schrödinger operators, with measures as potentials.

The basic result, from which everything else will follow, is Theorem 4.1 below. It says that the limits $W$ are necessarily reflectionless (this notion will be defined later) on the support of the absolutely continuous part of the spectral measure. This is a very strong condition; it severely restricts the structure of potentials with some absolutely continuous spectrum. As in [19], this result crucially depends on earlier work of Breimesser and Pearson $[7,8]$.

We will present two applications of Theorem 4.1 here; both are analogs of results from [19]. The first application gives an easy and transparent proof of a continuous Denisov-Rakhmanov [11, 12, 17] type theorem.

We denote by $\Sigma_{a c}$ the essential support of the absolutely continuous part of the spectral measure; this is determined up to sets of (Lebesgue) measure zero. If we write $\rho$ for the spectral measure, we can define (a representative of) $\Sigma_{a c}$ as the set where $d \rho / d t>0$. The absolutely continuous spectrum, $\sigma_{a c}$, may be obtained from $\Sigma_{a c}$ by taking the essential closure. The essential spectrum, $\sigma_{\text {ess }}$, can be defined as the set of accumulation points of the spectrum.

Theorem 1.1. Let $V$ be a uniformly locally integrable (half line) potential (that is, we assume that $\sup _{n} \int_{n}^{n+1}|V(x)| d x<\infty$ ). Suppose that

$$
\sigma_{e s s}=\Sigma_{a c}=[0, \infty)
$$

Then

$$
\lim _{x \rightarrow \infty} \int V(x+t) \varphi(t) d t=0
$$

for every continuous $\varphi$ of compact support.

Denisov proved this earlier [11, Theorem 2], under the somewhat stronger assumption that $V$ is bounded.

The conclusion of Theorem 1.1 says that $V(x)$ tends to zero as $x \rightarrow \infty$ in weak $*$ sense (more precisely, it is the sequence of measures $V(x+t) d t$ that converges). It will become clear later that this mode of convergence is natural here. Also, examples of the type $V=U^{2}+U^{\prime}$ with a rapidly decaying, but oscillating $U$ show that stronger modes of convergence of $V$ can not be expected.

Theorem 1.1 will be proved in Section 4. As in [19, Theorem 1.8], it should be possible to use the same technique to establish an analogous result for finite gap potentials and spectra (and beyond), but we will not pursue this theme here. 
Let us now discuss a second structural consequence of Theorem 4.1; in [19], I introduced the designation Oracle Theorem for statements of this type. The Oracle Theorem says that for operators with absolutely continuous spectrum, it is possible to approximately predict future values of the potential, with arbitrarily high accuracy, based on information about past values.

The precise formulation will involve measures $\mu$ as potentials and some additional technical devices; these will of course be explained in more detail later. To get a preliminary impression of what the Oracle Theorem is saying, it is possible to replace $\mu$ by a (uniformly locally integrable) potential $V$ in Theorem 1.2 below.

We will work with spaces $\mathcal{V}_{J}^{C}$ of signed Borel measures $\mu$ on intervals $J$. For now, we can pretend that a measure $\mu$ is in $\mathcal{V}_{J}^{C}$ if $|\mu|(J) \leq$ $C|J|$, but, for inessential technical reasons, the actual definition will be slightly different. If endowed with the weak $*$ topology, these spaces $\mathcal{V}_{J}^{C}$ are compact and in fact metrizable. The metric $d$ that is used below arises in this way. We will also use a similarly defined space $\mathcal{V}^{C}$ of measures on $\mathbb{R}$. See Section 2 for the precise definitions.

Finally, $S_{x} \mu$ will denote the shift by $x$ of the measure $\mu$, that is,

$$
\int f(t) d\left(S_{x} \mu\right)(t)=\int f(t-x) d \mu(t)
$$

If $d \mu=V d t$ is a locally integrable potential $V$, then this reduces to the shift map $\left(S_{x} V\right)(t)=V(x+t)$ that was introduced above.

Theorem 1.2 (The Oracle Theorem). Let $A \subset \mathbb{R}$ be a Borel set of positive (Lebesgue) measure, and let $\epsilon>0, a, b \in \mathbb{R}(a<b), C>0$. Then there exist $L>0$ and a continuous function (the oracle)

$$
\Delta: \mathcal{V}_{(-L, 0)}^{C} \rightarrow \mathcal{V}_{(a, b)}^{C}
$$

so that the following holds. If $\mu \in \mathcal{V}^{C}$ and the half line operator associated with $\mu$ satisfies $\Sigma_{a c} \supset A$, then there exists an $x_{0}>0$ so that for all $x \geq x_{0}$, we have that

$$
d\left(\Delta\left(\chi_{(-L, 0)} S_{x} \mu\right), \chi_{(a, b)} S_{x} \mu\right)<\epsilon .
$$

In other words, for large enough $x$, we can approximately determine the potential on $(x+a, x+b)$ from its values on $(x-L, x)$, and the function (oracle) that does this prediction is in fact independent of the potential. Moreover, by adjusting $a, b$, we can also specify in advance how far the oracle should look into the future. 


\section{TOPOLOGIES ON SPACES OF POTENTIALS}

We need a topology on a suitable set of potentials that makes this space compact and also interacts well with other basic objects such as $m$ functions. This is easy to do if we are satisfied with working with potentials that obey a local $L_{p}$ condition with $p>1$. Indeed, for every $p>1$ (and $C>0$ ), we can define

$$
\mathcal{V}_{p}^{C}=\left\{V: \mathbb{R} \rightarrow \mathbb{R}: \int_{n}^{n+1}|V(x)|^{p} d x \leq C^{p} \text { for all } n \in \mathbb{Z}\right\} .
$$

Closed balls in $L_{p}$ are compact in the weak $*$ topology if $p>1$; in fact, these compact topological spaces are metrizable. Pick such metrics $d_{n}$; in other words, if $W_{j}, W \in L_{p}(n, n+1),\left\|W_{j}\right\|_{p},\|W\|_{p} \leq C$, then $d_{n}\left(W_{j}, W\right) \rightarrow 0$ precisely if $W_{j} \rightarrow W$ in the weak $*$ topology, that is, precisely if

$$
\int_{n}^{n+1} W_{j}(x) g(x) d x \rightarrow \int_{n}^{n+1} W(x) g(x) d x \quad(j \rightarrow \infty)
$$

for all $g \in L_{q}(n, n+1)$, where $1 / p+1 / q=1$. Then, using these metrics, define, for $V, W \in \mathcal{V}_{p}^{C}$

$$
d(V, W)=\sum_{n=-\infty}^{\infty} 2^{-|n|} \frac{d_{n}\left(V_{n}, W_{n}\right)}{1+d_{n}\left(V_{n}, W_{n}\right)} ;
$$

here $V_{n}, W_{n}$ denote the restrictions of $V, W$ to $(n, n+1)$.

This metric generates the product topology on $\mathcal{V}_{p}^{C}$, where this space is now viewed as the product of the closed balls of radius $C$ in $L_{p}(n, n+$ $1)$. In particular, $\left(\mathcal{V}_{p}^{C}, d\right)$ is a compact metric space.

This simple device allows us to establish continuous analogs of the results of [19] without much difficulty at all, but it is unsatisfactory because the most natural and general local condition on the potentials is an $L_{1}$ condition. Since $L_{1}$ is not a dual space, we will then need to consider measures to make an analogous approach work. Thus we define

$$
\mathcal{V}^{C}=\{\mu \in \mathcal{M}(\mathbb{R}):|\mu|(I) \leq C \max \{|I|, 1\} \text { for all intervals } I \subset \mathbb{R}\} .
$$

Here, $\mathcal{M}(\mathbb{R})$ denotes the set of (signed) Borel measures on $\mathbb{R}$. We can now proceed as above to define a metric on $\mathcal{V}^{C}$ : Pick a countable dense (with respect to $\|\cdot\|_{\infty}$ ) subset $\left\{f_{n}: n \in \mathbb{N}\right\} \subset C_{c}(\mathbb{R})$, the continuous functions of compact support, and put

$$
\rho_{n}(\mu, \nu)=\left|\int f_{n}(x) d(\mu-\nu)(x)\right|
$$


Then define the metric $d$ as

$$
d(\mu, \nu)=\sum_{n=1}^{\infty} 2^{-n} \frac{\rho_{n}(\mu, \nu)}{1+\rho_{n}(\mu, \nu)} .
$$

Clearly, $d\left(\mu_{j}, \mu\right) \rightarrow 0$ if and only if

$$
\int f(x) d \mu_{j}(x) \rightarrow \int f(x) d \mu(x) \quad(j \rightarrow \infty)
$$

for all $f \in C_{c}(\mathbb{R})$. Moreover, $\left(\mathcal{V}^{C}, d\right)$ is a compact space. To prove this, let $\mu_{n} \in \mathcal{V}^{C}$. By the Banach-Alaoglu Theorem, closed balls in $\mathcal{M}([-R, R])$ are compact. Use this and a diagonal process to find a subsequence $\mu_{n_{j}}$ with the property that $\int f d \mu_{n_{j}} \rightarrow \int f d \mu$ for all $f \in C_{c}(\mathbb{R})$, for some $\mu \in \mathcal{M}(\mathbb{R})$. The proof can now be completed by noting that a measure $\nu \in \mathcal{M}(\mathbb{R})$ is in $\mathcal{V}^{C}$ if and only if

$$
\left|\int f(x) d \nu(x)\right| \leq C \max \{\operatorname{diam}(\operatorname{supp} f), 1\}\|f\|_{\infty}
$$

for all $f \in C_{c}(\mathbb{R})$.

The same construction can be run if $\mathbb{R}$ is replaced by an interval $J$, and these spaces, which we will denote by $\mathcal{V}_{J}^{C}$, will also play an important role later on.

\section{SCHRÖDINGER OPERATORS With MEASURES}

We are thus led to consider Schrödinger operators with measures as potentials; therefore, we must now clarify what the precise meaning of this object is. There is, of course, a considerable amount of previous work on these issues; see, for example, $[1,3,5,6]$ and the references cited therein. Here, we will follow the approach of [3]. Actually, Schrödinger operators will not play a central role in this paper, at least not explicitly. Therefore, we will only indicate how to make sense out of the Schrödinger equations $-f^{\prime \prime}+\mu f=z f$. We can then use these to define Titchmarsh-Weyl $m$ functions, spectral measures etc., and we refer the reader to [3] for the (straightforward) definition of domains that yield self-adjoint operators.

There are two obvious attempts, and these conveniently lead to the same result: If $I \subset \mathbb{R}$ is an open interval and $f \in C(I)$, we can call $f$ a solution to the Schrödinger equation

$$
-f^{\prime \prime}+f \mu=z f
$$


if (3.1) holds in the sense of distributions on $I$. Alternatively, one can work with the quasi-derivative

$$
(A f)(x)=f^{\prime}(x)-\int_{[0, x]} f(t) d \mu(t)
$$

if $x<0$, then $\int_{[0, x]}$ needs to be replaced with $-\int_{(x, 0)}$ here. We now say that $f$ solves (3.1) on $I$ if both $f$ and $A f$ are (locally) absolutely continuous and $-(A f)^{\prime}=z f$ on $I$. This new definition is motivated by the observation that, at least formally, $(A f)^{\prime}=f^{\prime \prime}-f \mu$.

A slight modification of the argument from the proof of $[3$, Theorem 2.4] then shows that this latter interpretation of (3.1) is equivalent to the equation holding in $\mathcal{D}^{\prime}(I)$. The basic observation here is that if $f$ is continuous, then $(A f)^{\prime}=f^{\prime \prime}-f \mu$ in $\mathcal{D}^{\prime}$, not only formally.

Note that if $f$ solves (3.1), then $f^{\prime}$ is of bounded variation and the jumps can only occur at the atoms of $\mu$.

If $\mu \in \mathcal{V}^{C}$, we have limit point case at both endpoints. This means that for $z \in \mathbb{C}^{+}$(the upper half plane in $\mathbb{C}$ ), there exist unique (up to a factor) solutions $f_{ \pm}(x, z)$ of $(3.1)$ on $\mathbb{R}$ satisfying $f_{-} \in L_{2}(-\infty, 0)$, $f_{+} \in L_{2}(0, \infty)$. The Titchmarsh-Weyl $m$ functions of the problems on $(-\infty, x)$ and $(x, \infty)$, with Dirichlet boundary conditions at $x(u(x)=$ $0)$, are now defined as follows:

$$
m_{ \pm}(x, z)= \pm \frac{f_{ \pm}^{\prime}(x, z)}{f_{ \pm}(x, z)}
$$

We will use this formula only for points $x$ with $\mu(\{x\})=0$ so that the possible discontinuities of $f^{\prime}$ cannot cause any problems here.

Definition 3.1. Let $A \subset \mathbb{R}$ be a Borel set. We call a potential $\mu \in \mathcal{V}^{C}$ reflectionless on $A$ if

$$
m_{+}(x, t)=-\overline{m_{-}(x, t)} \quad \text { for almost every } t \in A
$$

for some $x \in \mathbb{R}$ with $\mu(\{x\})=0$.

The set of reflectionless potentials $\mu \in \bigcup_{C>0} \mathcal{V}^{C}$ on $A$ is denoted by $\mathcal{R}(A)$.

This is a key notion for everything that follows. If we have (3.3) for some $x$, then we automatically get this equation at all points of continuity of $\mu$. Moreover, the exceptional set implicit in (3.3) can be taken to be independent of $x$. To prove these remarks, observe that if $m_{ \pm}(x, t) \equiv \lim _{y \rightarrow 0+} m_{ \pm}(x, t+i y)$ exists for some $x, t \in \mathbb{R}$, then this limit exists for all $x$ (and the same $t$ ). Moreover, as a function of $x$, the $m$ functions are of bounded variation and (using distributional 
derivatives)

$$
\pm \frac{d}{d x} m_{ \pm}=\mu-z-m_{ \pm}^{2}
$$

The claim now follows by considering $(d / d x)\left(m_{+}+\overline{m_{-}}\right)(x, t)$.

The other key notion is that of the $\omega$ limit set of a potential $\mu \in \mathcal{V}^{C}$ under the shift map. This was already mentioned in the introduction, and we can now give the more precise definition

$$
\omega(\mu)=\left\{\nu \in \mathcal{V}^{C}: \text { There exist } x_{n} \rightarrow \infty \text { so that } d\left(S_{x_{n}} \mu, \nu\right) \rightarrow 0\right\} .
$$

For the definition of the shifted measures $S_{x} \mu$, see (1.1). Typically, $\mu$ will be given as a half line potential $V$, but it is of course easy to interpret $V$ as an element $d \mu=V d x$ of $\mathcal{V}^{C}$ (such a $\mu$ automatically gives zero weight to $(-\infty, 0])$.

The compactness of $\mathcal{V}^{C}$ ensures that $\omega(\mu)$ is non-empty, compact, and invariant under $\left\{S_{x}: x \in \mathbb{R}\right\}$. Moreover, and in contrast to the discrete case, $\omega(\mu)$ is also connected because we now have a flow $S_{x}$.

\section{MAIN RESUlts AND THEIR PROOFS}

It will be useful to introduce $\mathcal{V}_{+}^{C}$ as the set of all $\mu \in \mathcal{V}^{C}$ with $|\mu|((-\infty, 0])=0$. These measures will serve as the potentials of half line problems on $(0, \infty)$. We can think of such a $\mu$ as a measure on $(0, \infty)$ or on $\mathbb{R}$.

Theorem 4.1. Let $\mu \in \mathcal{V}_{+}^{C}$. Then $\omega(\mu) \subset \mathcal{R}\left(\Sigma_{a c}\right)$.

Here, $\Sigma_{a c}$ denotes an essential support of the absolutely continuous part of the spectral measure of the half line problem on $(0, \infty)$ (say).

Theorem 4.1 is proved in the same way as the analogous result (Theorem 1.4) from [19]. Therefore, we will only make a few quick remarks and then leave the matter at that.

First of all, note that although the original result of Breimesser and Pearson [7, Theorem 1] is formulated for Schrödinger operators with locally integrable potentials, the same proof also establishes the result for operators with measures as potentials. Indeed, one never works with the potential itself but only with solutions to the Schrödinger equation (3.1) or with transfer matrices. See also [19, Appendix A].

As a second ingredient, we need continuous dependence of the (half line) $m$ functions $m_{ \pm}$on the potential.

Lemma 4.2. Let $\mu_{n}, \mu \in \mathcal{V}^{C}$ and suppose that $d\left(\mu_{n}, \mu\right) \rightarrow 0$. Fix $x \in \mathbb{R}$ with $\mu_{n}(\{x\})=\mu(\{x\})=0$. Then

$$
m_{ \pm}\left(x, z ; \mu_{n}\right) \rightarrow m_{ \pm}(x, z ; \mu),
$$

uniformly on compact subsets of $\mathbb{C}^{+}$. 
This follows because convergence in $\mathcal{V}^{C}$ implies weak $*$ convergence of the restrictions of the measures to compact intervals, at least if the endpoints of these intervals are not atoms of $\mu$. It then follows that the solutions to the Schrödinger equation converge, locally uniformly in $z$. This is most conveniently established by rewriting the Schrödinger equation as an integral equation. See, for example, [3, Lemma 6.3] for more details. One can now use (3.2) to obtain the Lemma. In fact, it is also helpful to approximate $m_{ \pm}$by $m$ functions of problems on bounded intervals. This allows us to work with solutions that satisfy a fixed initial condition.

To prove Theorem 4.1, fix $\nu \in \omega(\mu)$. By definition of the $\omega$ limit set, there exists a sequence $x_{j} \rightarrow \infty$ so that $S_{x_{j}} \mu \rightarrow \nu$ in $\left(\mathcal{V}^{C}, d\right)$. Fix $x \in \mathbb{R}$ with $\mu\left(\left\{x+x_{j}\right\}\right)=\nu(\{x\})=0$. By Lemma 4.2,

$$
m_{ \pm}\left(x+x_{j}, z ; \mu\right) \rightarrow m_{ \pm}(x, z ; \nu) \quad(j \rightarrow \infty),
$$

locally uniformly in $z$. The Breimesser-Pearson Theorem [7, Theorem 1] (see also [19, Theorem 3.1]) together with [19, Theorem 2.1] then yield a relation between $m_{+}(x, z ; \nu)$ and $m_{-}(x, z ; \nu)$ which turns out to be equivalent to the condition from Definition 3.1, with $A=\Sigma_{a c}$. This last part of the argument is identical with the corresponding treatment of [19].

Honesty demands that I briefly comment on a technical (and relatively insignificant point) here: To run the argument in precisely this form, one needs a slight modification of either Lemma 4.2 or the original Breimesser-Pearson Theorem. The easiest solution would be to prove the Breimesser-Pearson Theorem for two half line $m$ functions $m_{ \pm}$(in the original version from $[7,8], m_{-}$refers to a bounded interval). Alternatively, one can use a variant of Lemma 4.2 where the approximating $m$ functions may be associated with bounded (but growing) intervals.

Let us now show how Theorem 4.1 can be used to produce DenisovRakhmanov type theorems. We will automatically obtain the following slightly more general version of Theorem 1.1.

Theorem 4.3. Let $\mu \in \mathcal{V}_{+}^{C}$, and suppose that the half line operator generated by $\mu$ satisfies

$$
\sigma_{e s s}=\Sigma_{a c}=[0, \infty) .
$$

Then $d\left(S_{x} \mu, 0\right) \rightarrow 0$ as $x \rightarrow \infty$.

The proof will also depend on the following observation (whose discrete analog was pointed out in [15]; see also [16]). 
Proposition 4.4. Let $\mu \in \mathcal{V}_{+}^{C}$ and assume that $\nu \in \omega(\mu)$. Then

$$
\sigma(\nu) \subset \sigma_{\text {ess }}^{+}(\mu) \text {. }
$$

Here, $\sigma(\nu)$ is the spectrum of $-d^{2} / d x^{2}+\nu$ on $L_{2}(\mathbb{R})$, while $\sigma_{\text {ess }}^{+}(\mu)$ denotes the essential spectrum of the half line operator $-d^{2} / d x^{2}+\mu$ on $L_{2}(0, \infty)$ (say).

Proof. In the discrete case, this followed from a quick argument using Weyl sequences. In the continuous case, this device is not as easily implemented because of domain questions. The following alternative argument avoids these issues and thus seems simpler: Suppose that $d\left(S_{x_{j}} \mu, \nu\right) \rightarrow 0$. Then the whole line (!) operators associated with $S_{x_{j}} \mu$ converge in strong resolvent sense to the (whole line) operator generated by $\nu$. To prove this fact, one can argue as in Lemma 4.2 above.

Since the operators with shifted potentials are unitarily equivalent to the operator generated by $\mu$ itself, it follows from [18, Theorem VIII.24(a)] that $\sigma(\nu) \subset \sigma(\mu)$. The $\omega$ limit set does not change if $\mu$ is modified on a left half line; any discrete eigenvalue, however, can be moved (or removed) by such a modification. Similarly, $\sigma_{\text {ess }}=\sigma_{\text {ess }}^{+} \cup \sigma_{\text {ess }}^{-}$, and $\sigma_{e s s}^{-}$is completely at our disposal, so we actually obtain the stronger claim of the Proposition.

Proof of Theorem 4.3. We will show that $\omega(\mu)$ consists of the zero potential only. This will imply the claim because the distance between $S_{x} \mu$ and $\omega(\mu)$ must go to zero as $x \rightarrow \infty$.

By Theorem 4.1 and Proposition 4.4, any $\nu \in \omega(\mu)$ must satisfy

$$
\sigma(\nu)=[0, \infty), \quad \nu \in \mathcal{R}((0, \infty)) .
$$

Here, we use the (well known) fact that $\operatorname{Im} m_{ \pm}>0$ almost everywhere on $A$ if the corresponding potential is reflectionless on this set. Indeed, (3.3) shows that otherwise we would have $m_{+}+m_{-}=0$ on a set of positive measure, hence everywhere, but this is clearly impossible.

What we will actually prove now is that only the zero potential, $\nu=0$, satisfies (4.1). This argument follows a familiar pattern; see, for example, [9] or [21] (especially Lemma 4.6 and the discussion that follows) for similar arguments in somewhat different situations.

Suppose that $\nu \in \mathcal{V}^{C}$ obeys (4.1), and fix $x \in \mathbb{R}$ with $\nu(\{x\})=0$. Let $m_{ \pm}$be the $m$ functions of $H_{\nu}=-d^{2} / d t^{2}+\nu$ on $L_{2}(x, \infty)$ and $L_{2}(-\infty, x)$, respectively, and consider the function

$$
H(z)=m_{+}(x, z)+m_{-}(x, z)=-\frac{W\left(f_{+}, f_{-}\right)}{f_{+}(x, z) f_{-}(x, z)} .
$$


Here, $W(u, v)=u v^{\prime}-u^{\prime} v$ denotes the Wronskian. This last expression identifies $H$ as

$$
H(z)=-\frac{1}{G(x, x ; z)},
$$

the negative reciprocal of the (diagonal of the) Green function of $H_{\nu}$. Compare, for example, [10, Section 9.5]. (Of course, this reference does not discuss Schrödinger operators with measures, but the rather elementary argument based on the variation of constants formula generalizes without any difficulty.)

The defining property of $G$ is given by

$$
\left(\left(H_{\nu}-z\right)^{-1} \varphi\right)(x)=\int_{-\infty}^{\infty} G(x, y ; z) \varphi(y) d y .
$$

This holds for $z \notin \sigma(\nu)=[0, \infty), \varphi \in L_{2}(\mathbb{R})$. The spectral theorem shows that if $z=-t<0$, then

$$
\left\langle\varphi,\left(H_{\nu}+t\right)^{-1} \varphi\right\rangle=\int_{[0, \infty)} \frac{d\|E(s) \varphi\|^{2}}{s+t}>0 .
$$

Since $G$ is continuous in $x, y$, this implies that $G(x, x, t) \geq 0$, thus $H(t)<0$ for $t<0$. Furthermore, the fact that $\nu \in \mathcal{R}((0, \infty))$ implies that $\operatorname{Re} H(t)=0$ for almost every $t>0$.

So we know the phases of the boundary values of the Herglotz function $H$ almost everywhere. By the exponential Herglotz representation (or, synonymously, the Herglotz representation of $\ln H(z)$ ), this determines $H$ up to a (positive) multiplicative constant. Since $H_{0}(z)=$ $(-z)^{1 / 2}$ has the properties described above, this says that for suitable $c>0$,

$$
H(z)=c \sqrt{-z}=-\frac{c}{\sqrt{2}}+\frac{c}{\pi} \int_{0}^{\infty}\left(\frac{1}{t-z}-\frac{t}{t^{2}+1}\right) t^{1 / 2} d t .
$$

We will now need some information on the large $z$ asymptotics of $m$ functions. This subject has been analyzed in considerable depth; see, for example, $[2,13,14,20]$. Of course, the treatment of these references needs to be adjusted here to cover the case of Schrödinger operators with measures, but this is easy to do, especially since we will only need the rather unsophisticated estimate

$$
m_{ \pm}\left(x,-\kappa^{2}\right)=-\kappa+o(1) \quad(\kappa \rightarrow \infty) .
$$

Here, it is important that we assumed that $\nu(\{x\})=0$.

Since $H=m_{+}+m_{-}$, it now follows that $c=2$ in (4.2). Furthermore, the measures from the Herglotz representations of $m_{ \pm}$are absolutely 
continuous with respect to the measure

$$
d \rho(t)=\frac{2}{\pi} \chi_{(0, \infty)}(t) t^{1 / 2} d t
$$

from the Herglotz representation (4.2) of $H$. In fact, we can write

$$
m_{ \pm}(x, z)=A_{ \pm}+\int\left(\frac{1}{t-z}-\frac{t}{t^{2}+1}\right) g_{ \pm}(t) d \rho(t)
$$

with $A_{ \pm} \in \mathbb{R}, A_{+}+A_{-}=-\sqrt{2}$, and, more importantly, $0 \leq g_{ \pm} \leq 1$ and $g_{+}+g_{-}=1$. More can be said here: Since $\nu$ is reflectionless on $(0, \infty)$, we can use (3.3) to deduce that $\operatorname{Im} m_{+}(x, t)=\operatorname{Im} m_{-}(x, t)$ for almost every $t>0$. But for almost every $t>0$, we have that $\operatorname{Im} m_{ \pm}(x, t)=g_{ \pm}(t)(2 / \pi) t^{1 / 2}$, thus $g_{+}=g_{-}=1 / 2$ almost everywhere. It now follows that

$$
m_{ \pm}(x, z)=\sqrt{-z} .
$$

But $m_{0}=\sqrt{-z}$ is the $m$ function for zero potential, thus $\nu=0$, as desired. This last step is a basic result in inverse spectral theory for potentials ( $m$ determines $V$ ); here, we of course need a version for measures, but this extension poses no difficulties. See, for instance, $[3$, Theorem $6.2(\mathrm{~b})]$ (this needs to be combined with the fact that $m$ determines $\phi$, but this is also discussed in [3]).

It remains to prove the Oracle Theorem. We prepare for this by making a couple of new definitions. First of all, put

$$
\mathcal{R}^{C}(A)=\mathcal{R}(A) \cap \mathcal{V}^{C} \text {. }
$$

Next, we consider again spaces of half line potentials, and we now think of these as restrictions of measures $\mu \in \mathcal{V}^{C}$ :

$$
\begin{aligned}
& \mathcal{V}_{+}^{C}=\left\{\chi_{(0, \infty)} \mu: \mu \in \mathcal{V}^{C}\right\}, \\
& \mathcal{V}_{-}^{C}=\left\{\chi_{(-\infty, 0)} \mu: \mu \in \mathcal{V}^{C}\right\}
\end{aligned}
$$

I emphasize that on $\mathcal{V}_{ \pm}^{C}$, we do not use the topology that is induced by $\mathcal{V}^{C} \supset \mathcal{V}_{ \pm}^{C}$. That would quite obviously be a bad idea because it would make the restriction map $\mu \mapsto \chi_{(0, \infty)} \mu$ discontinuous; consider, for example, the sequence $\mu_{n}=\delta_{1 / n}$. Instead, we just observe that in the notation from Section 2, we can identify $\mathcal{V}_{+}^{C}=\mathcal{V}_{J}^{C}$, where $J=(0, \infty)$, and we use the topology and metric described in Section 2. In other words, if we denote this metric by $d_{+}$, then $d_{+}\left(\mu_{n}, \mu\right) \rightarrow 0$ if and only if

$$
\int f(x) d \mu_{n}(x) \rightarrow \int f(x) d \mu(x) \quad(n \rightarrow \infty)
$$

for all continuous $f$ whose support is a compact subset of $(0, \infty)$. Similar remarks apply to $\mathcal{V}_{-}^{C}$, of course. 
Now the restriction maps $\mathcal{V}^{C} \rightarrow \mathcal{V}_{ \pm}^{C}$ are continuous, and the spaces $\left(\mathcal{V}_{ \pm}^{C}, d_{ \pm}\right)$are compact.

Finally, we introduce

$$
\begin{aligned}
& \mathcal{R}_{+}^{C}(A)=\left\{\chi_{(0, \infty)} \mu: \mu \in \mathcal{R}^{C}(A)\right\} \subset \mathcal{V}_{+}^{C}, \\
& \mathcal{R}_{-}^{C}(A)=\left\{\chi_{(-\infty, 0)} \mu: \mu \in \mathcal{R}^{C}(A)\right\} \subset \mathcal{V}_{-}^{C},
\end{aligned}
$$

and we use the same metrics $d_{ \pm}$on these spaces also. With this setup, we now obtain statements that are analogs of [19, Proposition 4.1].

Proposition 4.5. Let $A \subset \mathbb{R}$ be a Borel set of positive measure, and fix $C>0$. Then:

(a) $\left(\mathcal{R}^{C}(A), d\right)$ and $\left(\mathcal{R}_{ \pm}^{C}(A), d_{ \pm}\right)$are compact spaces;

(b) The restriction maps

$$
\begin{aligned}
& \mathcal{R}^{C}(A) \rightarrow \mathcal{R}_{+}^{C}(A), \quad \mu \mapsto \chi_{(0, \infty)} \mu ; \\
& \mathcal{R}^{C}(A) \rightarrow \mathcal{R}_{-}^{C}(A), \quad \mu \mapsto \chi_{(-\infty, 0)} \mu
\end{aligned}
$$

are continuous and bijective (and thus homeomorphisms).

(c) The inverse map

$$
\mathcal{R}_{-}^{C}(A) \rightarrow \mathcal{R}^{C}(A), \quad \chi_{(-\infty, 0)} \mu \mapsto \mu
$$

is (well defined, by part (b), and) uniformly continuous.

Proof. (a) Since $\mathcal{R}^{C}(A)$ is a subspace of the compact space $\mathcal{V}^{C}$, it suffices to show that $\mathcal{R}^{C}(A)$ is closed. This can be done exactly as in [19, Proof of Proposition 4.1(d)]; we make use of Lemma 4.2 of the present paper and Theorem 2.1, Lemma 3.2 of [19].

The spaces $\mathcal{R}_{ \pm}^{C}(A)$ are the images of the compact space $\mathcal{R}^{C}(A)$ under the continuous restriction maps, so these spaces are compact, too.

(b) Continuity of the restriction maps is clear (and was already used in the preceding paragraph). Moreover, these maps are surjective by the definition of the spaces $\mathcal{R}_{ \pm}^{C}(A)$. Injectivity follows from eq. (3.3): $\mu$ on $(0, \infty)$ determines $m_{+}(x, \cdot)$ for all $x>0$. Fix an $x>0$ with $\mu(\{x\})=$ 0 . Since $\mu$ is reflectionless on $A$ and $|A|>0$, we have condition (3.3) on a set of positive measure, and this lets us find $m_{-}(x, \cdot)$. This $m$ function, in turn, determines $\mu$ on $(-\infty, x)$.

Finally, recall that a continuous bijection between compact metric spaces automatically has a continuous inverse.

(c) This is an immediate consequence of parts (a) and (b).

As in [19], the Oracle Theorem will follow by combining Proposition 4.5 with Theorem 4.1. In fact, in rough outline, things are rather obvious now: Proposition 4.5 says that a reflectionless potential can be approximately predicted if it is known on a sufficiently large interval 
(recall how the topologies on the spaces $\mathcal{R}^{C}(A), \mathcal{R}_{ \pm}^{C}(A)$ were defined), and Theorem 4.1 makes sure that $S_{x} \mu$ is approximately reflectionless for sufficiently large $x$.

Some care must be exercised, however, if a continuous oracle $\Delta$ is desired. The following straightforward but technical considerations prepare for this part of the proof. We again consider the spaces $\mathcal{V}_{J}^{C}$ with metrics of the type described in Section 2; in the applications below, the interval $J$ will be bounded and open, but this is not essential here.

In a normed space, balls $B_{r}(x)=\{y:\|x-y\|<r\}$ are convex; Lemma 4.6 below says that balls with respect to the metric (2.1) enjoy the following weaker, but analogous property.

Lemma 4.6. If $w_{j} \geq 0, \sum_{j=1}^{m} w_{j}=1$ and $\mu, \nu_{j} \in \mathcal{V}_{J}^{C}$ satisfy $d\left(\mu, \nu_{j}\right)<$ $\epsilon$ with $\epsilon \leq 1 / 4$ (say), then

$$
d\left(\mu, \sum_{j=1}^{m} w_{j} \nu_{j}\right)<6 \epsilon \ln \epsilon^{-1}
$$

Proof. Let $N=\max \left\{n \in \mathbb{N}: 2^{n+1} \epsilon \leq 1\right\}$, and abbreviate $\sum w_{j} \nu_{j}=\nu$. Since $d\left(\mu, \nu_{j}\right)<\epsilon$, it is clear from (2.1) that if $n \leq N$, then

$$
\rho_{n}\left(\mu, \nu_{j}\right)<\frac{2^{n} \epsilon}{1-2^{n} \epsilon} \leq 2^{n+1} \epsilon
$$

The definition of $\rho_{n}$ shows that

$$
\rho_{n}(\mu, \nu) \leq \sum w_{j} \rho_{n}\left(\mu, \nu_{j}\right)
$$

so we obtain that

$$
\rho_{n}(\mu, \nu)<2^{n+1} \epsilon \quad(n \leq N) .
$$

This allows us to estimate

$$
\sum_{n=1}^{N} 2^{-n} \frac{\rho_{n}(\mu, \nu)}{1+\rho_{n}(\mu, \nu)}<\sum_{n=1}^{N} 2^{-n} \cdot 2^{n+1} \epsilon=2 N \epsilon<\frac{2 \epsilon \ln \epsilon^{-1}}{\ln 2} .
$$

On the other hand, we of course have that

$$
\sum_{n>N} 2^{-n} \frac{\rho_{n}(\mu, \nu)}{1+\rho_{n}(\mu, \nu)}<\sum_{n>N} 2^{-n}=2^{-N}<4 \epsilon \leq \frac{4 \epsilon \ln \epsilon^{-1}}{\ln 4},
$$

so we obtain the Lemma.

Proof of Theorem 1.2. We begin by introducing some notation that will prove useful. Write

$$
J_{-}=(-L, 0), \quad J_{+}=(a, b) .
$$


Our goal is to (approximately) predict the restriction of $S_{x} \mu$ to $J_{+}$, and we are given the restriction of $S_{x} \mu$ to $J_{-}$. We will use subscripts + and - , respectively, for such restrictions. So, for example, $\nu_{+}=\chi_{J_{+}} \nu$, and this is now interpreted as an element of $\mathcal{V}_{J_{+}}^{C}$.

Next, note that although a metric is explicitly mentioned in Theorem 1.2 , by compactness, it suffices to establish the assertion for some metric that generates the weak $*$ topology. We will of course want to work with the metric from (2.1) and Lemma 4.6. More specifically, denote this metric (on $\mathcal{V}_{J_{+}}^{C}$ ) by $d_{+}$. We use a similar metric $d_{-}$on $\mathcal{V}_{J_{-}}^{C}$; on $\mathcal{V}^{C}$, we also fix such a metric $d$, but, in addition, we demand, as we may, that $d$ dominates $d_{ \pm}$in the following sense: If $\mu, \nu \in \mathcal{V}^{C}$, then

$$
d_{-}\left(\mu_{-}, \nu_{-}\right) \leq d(\mu, \nu), \quad d_{+}\left(\mu_{+}, \nu_{+}\right) \leq d(\mu, \nu) .
$$

(The same notation, $d_{ \pm}$, was used for different purposes in Proposition 4.5 ; since we are not going to explicitly use those metrics here, that should not cause any confusion.)

With these preparations out of the way, the proof can now be accomplished in four steps. Let $A \subset \mathbb{R},|A|>0, \epsilon>0, a, b \in \mathbb{R}(a<b)$, and $C>0$ be given.

Step 1: Use Proposition 4.5(c) and the definition of the topologies on $\mathcal{R}^{C}(A), \mathcal{R}_{-}^{C}(A)$ to find $L>0$ and $\delta>0$ such that the following holds: For $\nu, \widetilde{\nu} \in \mathcal{R}^{C}(A)$,

$$
d_{-}\left(\nu_{-}, \widetilde{\nu}_{-}\right)<5 \delta \quad \Longrightarrow \quad d_{+}\left(\nu_{+}, \widetilde{\nu}_{+}\right)<\epsilon^{2} .
$$

We further assume that $\delta \leq \epsilon$ here. (The suspicious reader will have noticed that it is at this point only that we can define $d_{-}$and $d$.)

Step 2: The set

$$
\mathcal{R}_{J_{-}}^{C}(A):=\left\{\mu_{-}: \mu \in \mathcal{R}^{C}(A)\right\}
$$

is compact by Proposition 4.5 (again, this is a continuous image of a compact space). Since $\mathcal{V}_{J_{-}}^{C}$ is compact, it follows that the closed $\delta$ neighborhood

$$
\bar{U}_{\delta}=\left\{\mu_{-} \in \mathcal{V}_{J_{-}}^{C}: d_{-}\left(\mu_{-}, \nu_{-}\right) \leq \delta \text { for some } \nu \in \mathcal{R}^{C}(A)\right\}
$$

is also compact. Therefore, there exist $\nu_{1}, \ldots, \nu_{N} \in \mathcal{R}^{C}(A)$ so that the $2 \delta$ balls about the $\nu_{j,-}$ cover $\bar{U}_{\delta}$. At these points, we can define a preliminary version of the oracle in the obvious way as

$$
\Delta_{0}\left(\nu_{j,-}\right)=\nu_{j,+}
$$

However, this will be modified in the next step. 
Step 3: We now define, for arbitrary $\sigma \in \bar{U}_{\delta}$,

$$
\Delta(\sigma)=\frac{\sum\left(3 \delta-d_{-}\left(\sigma, \nu_{j,-}\right)\right) \Delta_{0}\left(\nu_{j,-}\right)}{\sum\left(3 \delta-d_{-}\left(\sigma, \nu_{j,-}\right)\right)} .
$$

The sums are over those $j$ for which $d_{-}\left(\sigma, \nu_{j,-}\right)<3 \delta$. It's easy to see that $\Delta: \bar{U}_{\delta} \rightarrow \mathcal{V}_{J_{+}}^{C}$ is continuous. Moreover, if $j_{0} \in\{1, \ldots, N\}$ is such that $d_{-}\left(\sigma, \nu_{j_{0},-}\right)<2 \delta$, then $d_{-}\left(\nu_{j,-}, \nu_{j_{0},-}\right)<5 \delta$ for all $j$ contributing to the sum. Therefore, (4.4) shows that $d_{+}\left(\nu_{j,+}, \nu_{j_{0},+}\right)<\epsilon^{2}$ for these $j$. If $\epsilon>0$ was sufficiently small, then Lemma 4.6 now implies that

$$
d_{+}\left(\Delta(\sigma), \nu_{j_{0},+}\right)<6 \epsilon^{2} \ln \epsilon^{-2}<\epsilon,
$$

say. Recall that this holds for every $j_{0}$ for which $d_{-}\left(\sigma, \nu_{j_{0},-}\right)<2 \delta$. Moreover, for every $\sigma \in \bar{U}_{\delta}$, there is at least one such index $j_{0}$.

The oracle $\Delta$ has now been defined on $\bar{U}_{\delta}$, and this is all we need to do the prediction. However, if a (somewhat specious) continuous extension to all of $\mathcal{V}_{J_{-}}^{C}$ is desired, one can proceed as above, by considering a suitable covering and taking convex combinations. It is also possible, somewhat more elegantly, to just refer to the extension theorem of Dugundji-Borsuk [4, Ch. II, Theorem 3.1].

Step 4: In this final step, we show that $\Delta$ indeed predicts $\mu$. Given a potential $\mu \in \mathcal{V}^{C}$ with $\Sigma_{a c} \supset A$, first of all take $x_{0}$ so large that

$$
d\left(S_{x} \mu, \omega(\mu)\right)<\delta \quad \text { for all } x \geq x_{0} .
$$

In other words, if we fix $x \geq x_{0}$, we then have that

$$
d\left(S_{x} \mu, \nu\right)<\delta
$$

for some (in general: $x$ dependent) $\nu \in \omega(\mu)$. By Theorem 4.1, $\nu \in$ $\mathcal{R}^{C}(A)$. When we restrict to $J_{ \pm}$, then (4.3), (4.6) imply that

$$
d_{-}\left(\left[S_{x} \mu\right]_{-}, \nu_{-}\right)<\delta, \quad d_{+}\left(\left[S_{x} \mu\right]_{+}, \nu_{+}\right)<\delta .
$$

In particular, this ensures that $\left[S_{x} \mu\right]_{-} \in \bar{U}_{\delta}$, and thus there exists a $j \in\{1, \ldots, N\}$ so that

$$
d_{-}\left(\left[S_{x} \mu\right]_{-}, \nu_{j,-}\right)<2 \delta
$$

By (4.5),

$$
d_{+}\left(\Delta\left(\left[S_{x} \mu\right]_{-}\right), \nu_{j,+}\right)<\epsilon .
$$

But by the triangle inequality, we also have that $d_{-}\left(\nu_{-}, \nu_{j,-}\right)<3 \delta$, so (4.4) shows that

$$
d_{+}\left(\nu_{+}, \nu_{j,+}\right)<\epsilon^{2} .
$$

If this is combined with (4.7), (4.8), we indeed obtain that

$$
d_{+}\left(\Delta\left(\left[S_{x} \mu\right]_{-}\right),\left[S_{x} \mu\right]_{+}\right)<\delta+\epsilon+\epsilon^{2}<3 \epsilon
$$


(say), as desired.

Acknowledgment. I thank Sergey Denisov and Barry Simon for bringing [11] to my attention and Lenny Rubin for useful information on extension theorems.

\section{REFERENCES}

[1] S. Albeverio, F. Gesztesy, R. Hoegh-Krohn, and H. Holden, Solvable Models in Quantum Mechanics, Texts and Monographs in Physics, Springer-Verlag, New York, 1988.

[2] F.V. Atkinson, On the location of the Weyl circles, Proc. Royal Soc. Edinburgh Sect. A88 (1981), 345-356.

[3] A. Ben Amor and C. Remling, Direct and inverse spectral theory of Schrödinger operators with measures, Int. Eq. Op. Theory 52 (2005), 395-417.

[4] C. Bessaga and A. Pelczynski, Selected Topics in Infinite-Dimensional Topology, Mathematical Monographs, Vol. 58, Polish Scientific Publishers, Warsaw, 1975.

[5] J.F. Brasche, P. Exner, Y.A. Kuperin, and P. Seba, Schrödinger operators with singular interactions, J. Math. Anal. Appl. 184 (1994), 112-139.

[6] J.F. Brasche, R. Figari, and A. Teta, Singular Schrödinger operators as limits of point interaction Hamiltonians, Potential Anal. 8 (1998), 163-178.

[7] S.V. Breimesser and D.B. Pearson, Asymptotic value distribution for solutions of the Schrödinger equation, Math. Phys. Anal. Geom. 3 (2000), 385-403.

[8] S.V. Breimesser and D.B. Pearson, Geometrical aspects of spectral theory and value distribution for Herglotz functions, Math. Phys. Anal. Geom. 6 (2003), 29-57.

[9] S. Clark, F. Gesztesy, H. Holden, and B.M. Levitan, Borg-type theorems for matrix-valued Schrödinger operators, J. Differential Equations 167 (2000), 181-210.

[10] E.A. Coddington and N. Levinson, Theory of Ordinary Differential Equations, McGraw-Hill, New York, 1955.

[11] S. Denisov, On the continuous analog of Rakhmanov's theorem for orthogonal polynomials, J. Funct. Anal. 198 (2003), 465-480.

[12] S. Denisov, On Rakhmanov's theorem for Jacobi matrices, Proc. Amer. Math. Soc. 132 (2004), 847-852.

[13] F. Gesztesy and B. Simon, A new approach to inverse spectral theory. II. General real potentials and the connection to the spectral measure, Ann. of Math. 152 (2000), 593-643.

[14] D.B. Hinton, M. Klaus, and J.K. Shaw, Series representation and asymptotics for Titchmarsh-Weyl $m$-functions, Differential Integral Eq. 2 (1989), 419-429.

[15] Y. Last and B. Simon, Eigenfunctions, transfer matrices, and absolutely continuous spectrum of one-dimensional Schrödinger operators, Invent. Math. 135 (1999), 329-367.

[16] Y. Last and B. Simon, The essential spectrum of Schrödinger, Jacobi, and CMV operators, J. Anal. Math. 98 (2006), 183-220.

[17] E.A. Rakhmanov, The asymptotic behavior of the ratio of orthogonal polynomials II (Russian), Mat. Sb. (N.S.) 118(160) (1982), 104-117. 
[18] M. Reed and B. Simon, Methods of modern mathematical physics, I. Functional analysis, Academic Press, New York, 1980.

[19] C. Remling, The absolutely continuous spectrum of Jacobi matrices, preprint, http://arxiv.org/abs/0706.1101

[20] A. Rybkin, Some new and old asymptotic representations of the Jost solution and the Weyl $m$-function for Schrödinger operators on the line, Bull. London Math. Soc. 34 (2002), 61-72.

[21] R. Sims and G. Stolz, Localization in one-dimensional random media: a scattering theoretic approach, Comm. Math. Phys. 213 (2000), 575-597.

Mathematics Department, University of Oklahoma, Norman, OK 73019

E-mail address: cremling@math.ou.edu

$U R L$ : www . math.ou.edu/ cremling 